Caja Costaricense de Seguro Social

Hospital San Juan de Dios

Servicio de Farmacia

Centro de Información Medicamentos
Agosto 2015

Número 8, Volumen 5

\title{
Hepatotoxicidad por medicamentos
}

\section{Introducción}

La mayoría de las reacción de metabolización se producen en el hígado, lo que determina que sea el principal órgano diana de posibles reacciones adversas (RAM).

La hepatotoxicidad es un daño al hígado producido por una disfunción del órgano causada por la exposición a un medicamento u otro agente no infeccioso. Son especialmente preocupantes las reacciones adversas serias que podrían resultar en condiciones incapacitantes, generar secuelas permanentes o bien requerir hospitalización.

Aunque es considerable el número de fármacos que se han relacionado con hepatoxicidad, se considera una RAM de baja frecuencia. Para muchos principios activos se estima que la incidencia varía entre 1 en 10000 o 1 en 100000 pacientes. Debido a esta baja frecuencia, es posible que no sea detectada durante los ensayos clínicos previo a la comercialización del medicamento.

La incidencia real del problema es difícil de establecer debido al subregistro de los casos, dificultad en el diagnóstico, entre otras causas.

En la mayoría de los casos, no existe un tratamiento o antídoto para revertir la hepatotoxicidad, ante una reacción adversa de este tipo, se sugiere suspender el tratamiento.

El uso de $\mathrm{N}$-acetilcisteína después de una sobredosis de acetaminofén y el uso de carnitina intravenosa para el daño mitocondrial inducido por valproato, son solo algunas excepciones al uso de antídotos para prevenir toxicidad hepática.

Se estima que en Estados Unidos, la hepatotoxicidad por medicamentos es la primera causa de insuficiencia hepática aguda entre los pacientes referidos a un programa de trasplante hepático. Además según estadísticas de la FDA, la intoxicación por acetaminofén continua siendo la primera causa de insuficiencia hepática aguda en pacientes sin antecedente de enfermedad hepática previa.

La hepatotoxicidad es uno de los motivos más frecuentes de retirada de fármacos del mercado, por este motivo en este boletín se realiza una revisión de la hepatotoxicidad producida por esta causa.

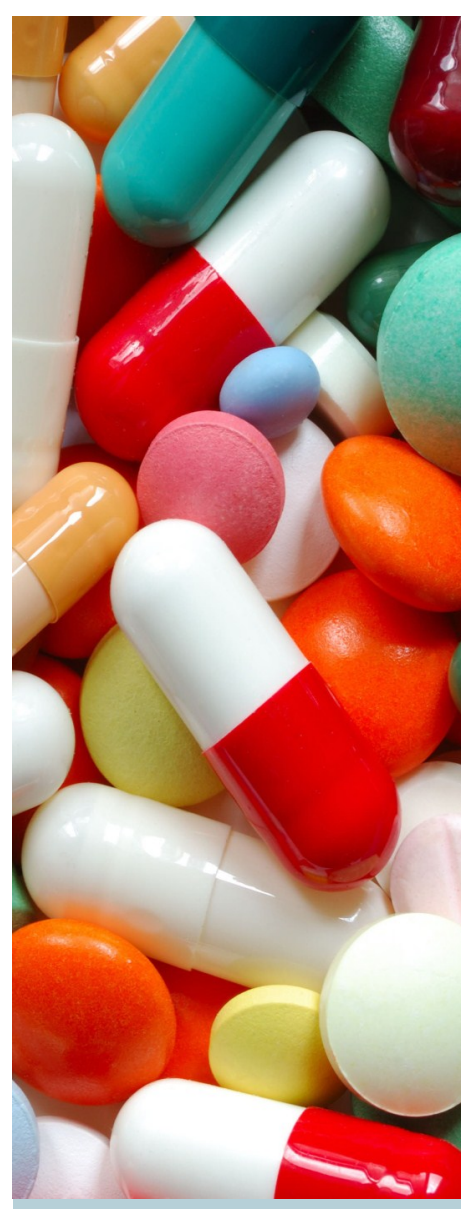

La incidencia real del problema (hepatotoxicidad por medicamentos) es dificil de establecer debido al subregistro de los casos, dificultad en el diagnóstico, entre otras causas 


\section{Lesión hepática y sus características}

Un panel de expertos de Estados Unidos y Europa en 1989 definió por conceso el concepto de lesión hepática, posteriormente dicho concepto fue retomado en el 2001 por una conferencia liderada por la el Centro de Evaluación e Investigación de Medicamentos de la FDA, Asociación de Investigación y Manufactura farmacéutica y por la Asociación para el Estudio de las Enfermedades Hepáticas.

De esta manera, se estableció que la lesión hepática se define como un aumento de más de tres veces el límite superior normal de alanino aminotransferasa (ALT), más de dos veces el límite superior normal de fofatasa alcalina o una bilirrubina total aumentada más de veces el límite superior normal asociada a cualquier elevación en la alanino aminotransferasa o fosfatasa alcalina.
La lesión hepática puede ser hepatocelular cuando existe una elevación inicial predominante de los niveles de alanino aminotransferasa o puede ser colestásica cuando se presenta una la elevación inicial predominante de fosfatasa alcalina.

El reconocer las características de la lesión hepática puede ayudar a categorizarlas. Según el tipo de medicamento, algunos podrían mostrar un patrón hepatocelular o colestásico. Estas categorías de lesión hepática no son mutualmente excluyentes e inclusive podrían encontrarse un patrón mixto.

Un artículo publicado en New England Journal of Medicine proporciona una serie de ejemplos de medicamentos que pueden inducir a una lesión hepática de tipo hepatocelular, colestásica o mixta (Ver tabla \#1).

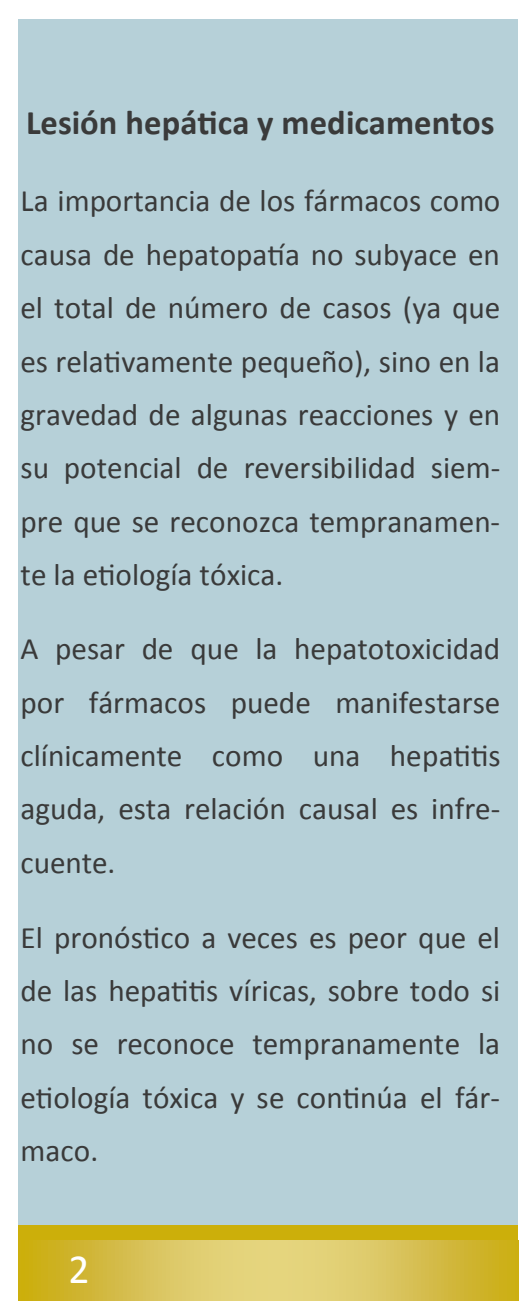

\section{Tabla \#1 Diferentes patrones de daño hepático}

\section{Hepatocelular \\ (ALT elevada)}

Acarbosa

Acetaminofén

Ácido valproico

AINES

Alopurinol

Amiodarona

Buproprion

Estatinas

Fluoxetina

Tratamiento antiretroviral

Isoniazida

Ketoconazol

Lisinopril

Losartán

Metotrexate

Omeprazol

Paroxetina

Pirazinamida

Rifampicina

Risperidona

Sertralina

Tetracicilinas
Mixta

(ALT + Fosfatasa alcalina aumentadas)

Amitriptilina

Azatioprina

Captopril

Carbamazepina

Clindamicina

Enalapril

Flutamida

Nitrofurantoína

Fenobarbital

Fenitoína

Sulfonamidas

Trimetoprim-Sulfametoxazol

Verapamilo
Colestásica

(Fosfatasa alcalina y Bilirrubina total aumentas)

Amoxicilina-Ácido Clavulánico Anticonceptivos orales

Antidepresivos tricíclicos

Eritromicina

Esteroides anabólicos

Estrógenos

Clorpromazina

Clopidogrel

Irbesartán

Mirtazapina

Terbinafina 


\section{Daño hepático versus lesión hepática}

La lesión hepática generalmente se establece cuando existe una elevación en los niveles de transaminasas séricas, aumentos de más de tres veces el límite superior normal, podrían no generar daño hepático.

Lo anterior se debe a la gran capacidad que tiene el hígado para remediar esa lesión, con el subsecuente desarrollo de tolerancia adaptativa, como se logra observer con la exposición inicial de medicamentos como la isoniazida.

La alteración de estas pruebas hepaticas no necesariamente representan o predicen la presencia de hepatotoxicidad.

Signos y síntomas como fatiga, anorexia, nausea, malestar en cuadrante superior derecho y orina oscura podrían ser indicativos de inicio de hepatotoxicidad.

Se podría considerer hepatotoxidad por medicamentos cuando aparezcan estos síntomas en conjunto con evidencia bioquímica de lesión hepática.

\section{Factores de susceptibilidad y mecanismos de hepatotoxicidad}

1. Factores genéticos: determinan la actividad de las vías metabólicas, la efectividad de los factores protectores del organismo y la regulación de la respuesta immune. La base genética puede condicionar diferencias étnicas en cuanto a susceptibilidad para determinados fármacos, así como la afectación de varios miembros de la misma familia. La existencia de polimorfismos genéticos de los CYP y enzimas hepaticas determina una variabilidad individual en el metabolism de los fármacos que puede conducir a la aparición de hepatotoxicidad.

2. Edad: la incidencia de reacciones adversas es de dos a tres veces más elevada en los adultos mayores y se atribuye a la prescripción, al uso concomitante de mútiples fármacos y a los efectos indirectos de enfermedades concomitants. El riesgo se asocial a reacciones dependientes de la dosis, debido a alteraciones en la biodisponibilidad y en el metabolism de fármacos. Además, la gravedad de la lesión hepática por medicamentos también parece aumentar con la edad. La hepatotoxicidad es mucho más rara en niños ya que metabolizan los fármacos con mayor rapidez debido al increment de las vías metabólicas oxidativas, aunque presentan una susceptibilidad aumentada a los salicitados.

3. Sexo: la frecuencia de hepatotoxicidad con expression clínica de hepatitis aguda es mayor en las mujeres, así como la susceptibilidad para los fármacos que causan hepatitis crónica activa. El riesgo es mayor en mujeres en edad próxima a la menopausia y se cree que los factores hormonales podrían modular el sistema immune en el sentido de predisponer a sufrir hepatotoxicidad. En el sexo masculino son más frecuentes las alteraciones vasculares de la azatioprina, especialmente tras un trasplante renal.

4. Estado nutricional: el ayuno aumenta la actividad del CYP2E1 a la vez que reduce los valores de glutatión y ambos factores parecen contribuir al mayor riesgo de hepatotoxicidad por acetaminophen en alcohólicos.

5. Antecedentes de reacción adversa a fármacos: aumentan el riesgo de reacción a otros fármacos explicándose por una possible predisposición genética a sufrir una respuesta inmunológica; también pueden existir reacciones cruzadas entre medicamentos de la misma familia.

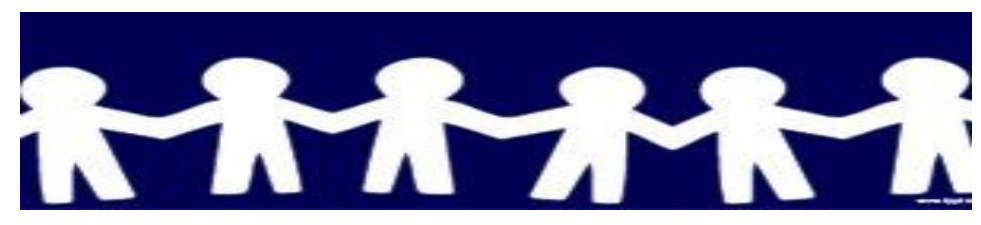




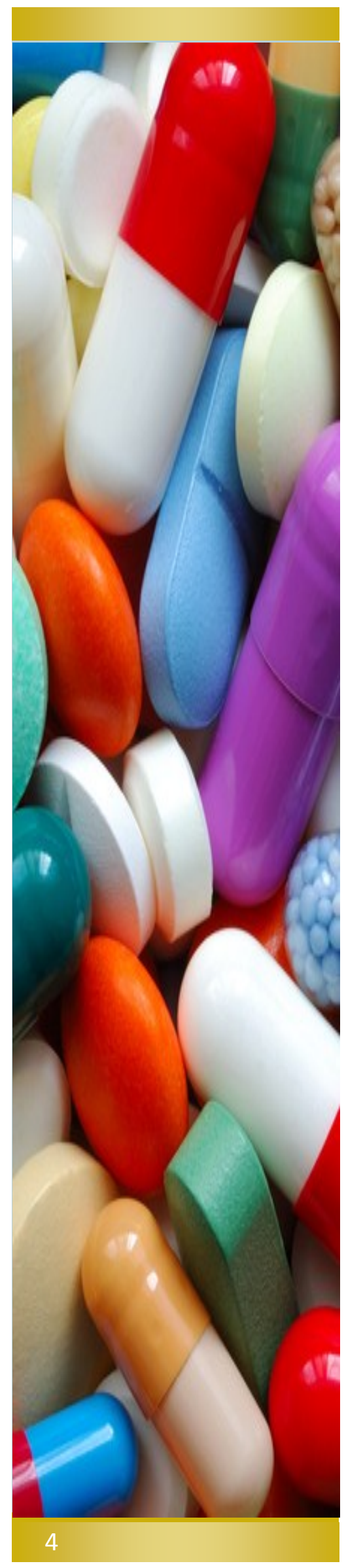

\section{Factores de susceptibilidad y mecanismos de hepatotoxicidad}

\section{Enfermedades concomitantes: el}

Hiper o hipotiroidismo se asocian a posibles alteraciones del aclaramiento hepático de los fármacos, habiéndose descrito también mayor riesgo de hepatotoxicad en los diabéticos. Los enfermos con lupus eritematoso sistémico (LES) o artitis reumatoide juvenile tienen una predisposición especial a sufrir hepatotoxicidad por salicilatos, al igual que está aumentando el riesgo para distintos medicamentos en pacientes con enfermedad inflamatoria intestinal o con insuficiencia renal.

Hepatopatía previa: interfiere en el metabolism de muchos medicamentos o altera su farmacodinamia. Sin embargo, una reacción hepatotóxica es más difícil de identificar en estos casos, cuando el deterioro de la function hepática puede ser un fenómeno evolutivo de la enfermedad de base. La hepatotoxicidad puede comportar una gravedad desproporcionada en los pacientes con cirrosis.

\section{Mecanismos patogénicos de la hepa- totoxicidad}

Los fármacos que generan lesión hepática pueden actuar como:

a. hepatotoxinas intrínsecas, es decir produciendo toxicidad predecible, dependiente de la dosis y reproducible o

b. b. hepatotoxinas idiosincráticas, originándose toxicidad no predecible, no dependiente de la dosis ni reproducible.
Hepatotoxinas intrínsecas: suelen causar lesión a través de sus metabolites tóxicos, que pueden ser radicales libres, moléculas electrofílicas u oxígeno activo. Puede originar un mecanismo directo o indirecto

A. La hepatotoxicidad directa implica una alteración de la membrana celular y de las organelas a través de la peroxidación de lípidos de membrana, causando la necrosis hepatocitaria. El daño es usualmente citolítico, pero puede ser colestásico si la necrosis afecta principalmente al epitelio biliar.

B. Las hepatotoxinas que producen daño por un mecanismo indirecto ocasionan distorción de moléculas esenciales o el bloqueo selectivo de vías metabólicas fundamentals para la integridad celular. El daño hepático puede ser citotóxico (necrosis o esteatosis) o colestásico (interferencia selective con la excreción de sustancias a los canalículos biliares.

Hepatotoxidad idiosincrática: producen lesión hepática de forma impredecible no ligada a la dosis. Afecta tan solo a una pequeña proporción de pacientes tratados y puede estar mediada por idiosincrasia inmunológica o metabólica. La inmunológica depende de un periodo de hipersensibilidad tras una exposición fija una a de 5 semanas, asociado a fiebre, ictericia, eosinofilia 


\section{Diagnóstico de la hepatotoxicidad por fármacos}

Es un reto difficl en la práctica clínica diaria. Se considera que la lesión hepática por fármacos está infradiagnosticada por el bajo nivel de sospecha clínica y porque el diagnostico diferencial es habitualmente problemático.

Es necesario identificar el fármaco causante en caso de que más de uno esté bajo sospecha, ya que se pueden prevenir la evolución a hepatopatías más graves o crónicas (incluyendo fallo hepático fulminante) y evitar la recurrencia.

La ausencia de pruebas diagnósticas específicas, junto al hecho de que la reexposición dirigida rara vez está justificada por constituir un riesgo in-

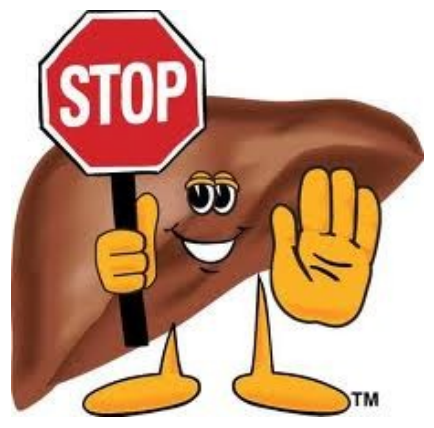
aceptable, representa un problema para alcanzar un diagnostic de certeza.

\section{"El diagnóstico de la hepatotoxicidad por fármacos es un reto difícil en la práctica clínica diaria."}

\section{Tratamiento de la hepatopatía por fármacos}

Se puede afirmar que en general, el tratamiento de la lesión hepática producida por fármacos es insatisfactorio. La excepción es el acetaminofén, para el que se dispone de un antidoto. La medida más importante consiste en la suspension inmediata del fármaco sospechoso de producir daño hepático. Puede resultar problemático cuando se administran varios medicamentos a la vez; en estos casos, la norma se basa en considerar al que se haya introducido más recientemente como el posible agente etiológico o en su defecto a aquel o aquellos que previamente hayan sido reconocidos como potencialmente hepatotóxico.

Si el medicamento es una hepatotoxina dependiente de la dosis, se tomarán las medidas oportunas para su eliminación del organism en relación con el tiempo transcurrido desde la toma del fármaco.
Cuando la reacción tóxica es intensea y se acompaña de síntomas graves, puede ser necesario el ingreso hospitalario del paciente y la aplicación de un tratamiento de soporte adecuado

Se debe presentar especial atención a un correcto balance hidroelectrolítico y nutricional, tratando los trastornos de la coagulación.

Hay que tener presente que mediamentos como la isoniazida y las estatinas pueden causar una elevación de las enzimas hepaticas de forma temporal con un curso asintomática, lo cual es normal y forma parte de los mecanismo de adaptación.

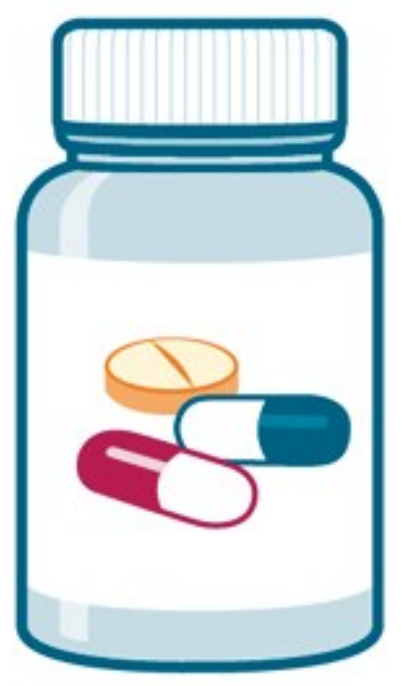




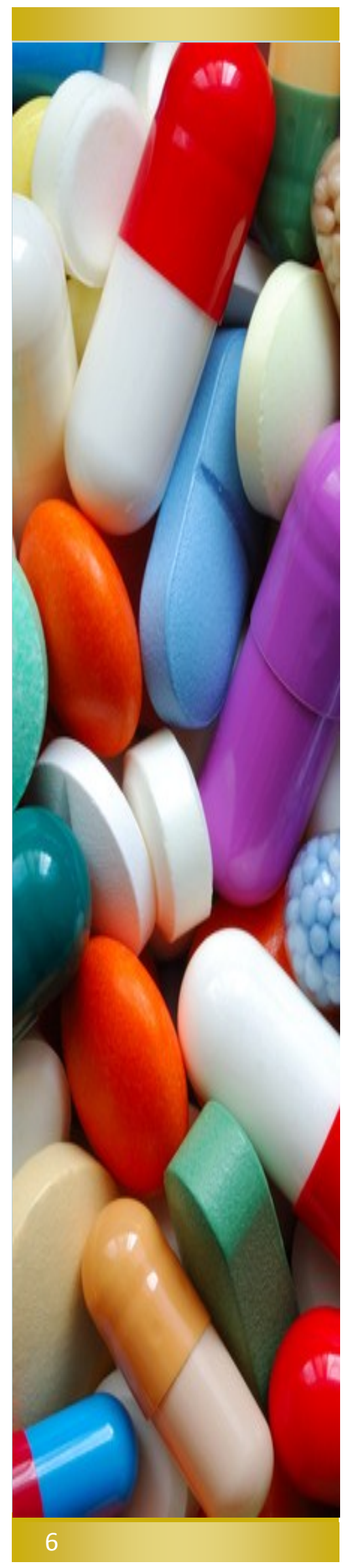

\section{Estrategias de Prevención}

1. Durante el desarrollo de medicamentos: es la primera opotunidad para prevenir hepatotoxicidad. Los estudios pre-clínicos son más útiles para detectar hepatoxicidad dosis dependiente que los estudios realizados en humanos. Los estudios fase 1 son la primera oportunidad para identificar hepatotoxicidad en humanos.

2. Farmacovigilancia: esta es la principal herramienta para detectar hepatotoxicidad postcomercialización en condiciones reales de uso, principalmente útil cuando se trata de un evento de baja frecuencia.

\section{Aletas de Farmacovigilancia}

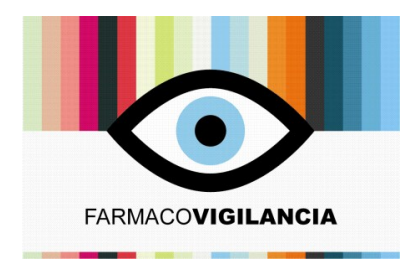

A continuación un resumen de las alertas de Farmacovigilancia más importantes emitidas por las agencias reguladoras internacionales.

\section{Fingolimod}

La FDA advierte sobre el reporte de un caso de leucoencefalopatía multifocal progresiva (LPM) definitiva y de un caso de probable LPM en pacientes que tomaban fingolimod por la esclerosis múltiple (EM).

Se trata de los primeros casos de LPM reportados en pacientes que toman este medicamentos, a quienes no se les había tratado previamente con inmunodepresores para la esclerosis múltiple (EM) ni para ninguna otra afección médica. Como consecuencia, se ha incorporado a la etiqueta del medicamento la información relacionada con estos casos recientes.

http://www.fda.gov/Drugs/DrugSafety/ucm457503.htm, 04 de agosto 2015

\section{Abiraterona}

La Agencia Japonesa del Medicamento y el Ministerio de Salud japonés, informa sobre los cambios que han introducido al etiquetado del medicamento debido a los reportes recibidos de insuficiencia hepática y hepatitis fulminante

http://www.who.int/medicines/publications/Pharm_Newsletter4_2015.pdf?ua=1, boletín julio-agosto 


\begin{abstract}
Alertas de Farmacovigilancia
3. Denosumab: la agencia de medicamentos y productos de interés sanitario briánico, emitió un comunicado de seguridad acerca del potencial de este medicamento de causar osteonecrosis mandibular. El énfasis de la comunicación surge como producto de una revision bibliográfica sobre el tema que realize la agencia.

http://www.who.int/medicines/publications/Pharm_Newsletter4_2015.pdf? ua $=1$, boletín Julio-agosto
\end{abstract}

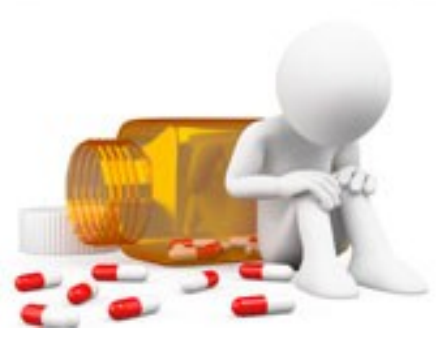

\title{
Bibliografía
}

1. Navarro, V, Senior, J. Drug-Related Hepatotoxicity. New England Journal of Medicine 2006; 354:731-9

2. Moreno, R. Hepatotoxicidad por fármacos. Revista Española de Reumatología Suplementos. Volumen 1. Número 1, 2002

3. http://www.uptodate.com.ezproxy.sibdi.ucr.ac.cr:2048/contents/drug-induced-liver-injury? source=search_result\&search=hepatotoxidad+por+medicamentos\&selectedTitle=1 150, consultado el 25-0915

\section{Hospital San Juan de Dios Servicio de Farmacia Centro de Información de Medicamentos}

Elaborado por: Dra. Cristina Fernández Barrantes, Farmacéutica Consultas:

- Teléfono: 2547-8324

- Correo electrónico: cimf_hsjd@ccss.sa.cr

Puede encontrar números anteriores del boletín en:

Revista Clínica- Hospital San Juan de Dios/ Escuela de Medicina UCR

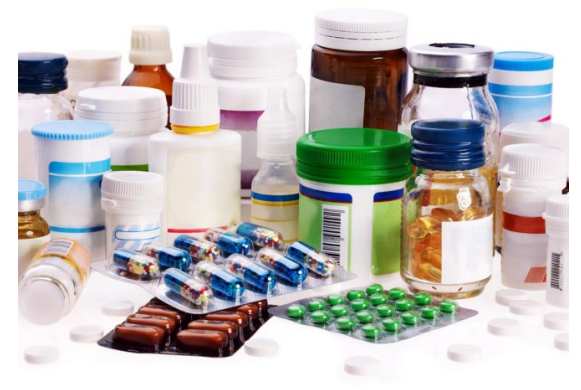

